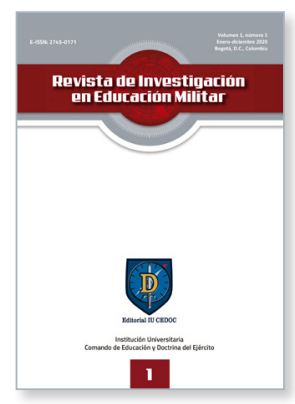

Revista de Investigación en Educación Militar

Volumen 1, número 1, enero-diciembre 2020, pp. 69-91

Bogotá, D. C., Colombia

E-ISSN: 2745-0171

https://doi.org/10.47961/27450171.7

\title{
La robótica creativa para el desarrollo de la cultura Maker inclusiva en la enseñanza fundamental: caso Escuela Municipal de Capistrano de Abreu, en São Paulo, Brasil
}

\section{Andrezza da Costa Rodrigues Basile}

https://orcid.org/0000-0002-1411-4864

andrezza.rodrigues@sme.prefeitura.sp.gov.br

Secretaria Municipal da Educación de São Paulo, Brasil

\section{Felipe Rodrigues Martinez Basile}

https://orcid.org/0000-0002-0404-4807

felipe.basile@ifsp.edu.br

Instituto Federal de Educação, Ciência e Tecnologia de São Paulo - Campus São Paulo Pirituba, Brasil

\section{Leonardo Juan Ramírez López}

https://orcid.org/0000-0002-6473-5685

leonardo.ramirez@unimilitar.edu.co

Universidad Militar Nueva Granada, Bogotá D. C., Colombia

Citación: Da Costa Rodrigues Basile, A., Rodrigues Martinez Basile, F., \& Ramírez López, L. J. (2020). La robótica creativa para el desarrollo de la cultura Maker inclusiva en la enseñanza fundamental: caso Escuela Municipal de Capistrano de Abreu, en São Paulo, Brasil. Revista de Investigación en Educación Militar, 1(1), 69-91. DOI: https://doi.org/10.47961/27450171.7

Publicado en línea: $1 .^{\circ}$ de diciembre de 2020

Los artículos publicados por la Revista de Investigación en Educación Militar son de acceso abierto bajo una licencia Creative Commons: Atribución - No Comercial - Sin Derivados.

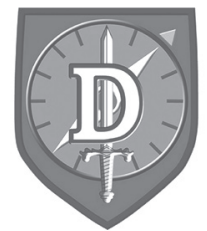

Editorial IU CEDOC 


\title{
La robótica creativa para el desarrollo de la cultura Maker inclusiva en la enseñanza fundamental: caso Escuela Municipal de Capistrano de Abreu, en São Paulo, Brasil
}

\author{
Creative robotics for the development of inclusive \\ Maker culture in elementary education: the case of the \\ Capistrano de Abreu Municipal School, in São Paulo, Brazil
}

\author{
Andrezza da Costa Rodrigues Basile \\ Secretaria Municipal de Educação de São Paulo, Brasil \\ Felipe Rodrigues Martinez Basile \\ Instituto Federal de Educação, Ciência e Tecnologia de São Paulo - Campus São Paulo Pirituba, Brasil \\ Leonardo Juan Ramírez López \\ Universidad Militar Nueva Granada, Bogotá, D. C., Colombia
}

\section{Resumen}

Este trabajo sobre la robótica creativa presenta un estudio de caso en el que se integra la cultura inclusiva Maker al aprendizaje en la institución de educación básica Capistrano de Abreu, en São Paulo, Brasil. Se presentan las estrategias y acciones pedagógicas basadas en la planificación didáctica y la formación para los profesores. Los resultados y aportes de este estudio hacen sugerencias para que los educadores y los sistemas escolares logren la inclusión digital a través de la robótica creativa, el movimiento Maker y elementos del Design Thinking. Estas herramientas educativas promueven el protagonismo del estudiante y potencian sus capacidades en medio de una comunidad necesitada, que en su mayoría tiene poco acceso a la tecnología de la información y la comunicación digital en sus contextos sociales.

Palabras clave: aprendizaje; cultura Maker; Design Thinking; inclusión digital; programación visual; robótica.

\section{Abstract}

This analysis on creative robotics presents a case study in which the inclusive culture Maker is integrated into learning at the Capistrano de Abreu basic education institution, in São Paulo, Brazil. The strategies and educational actions based on educational planning and training for teachers are disclosed. The results and contributions of this study make suggestions for educators and school systems to achieve digital inclusion through creative robotics, the Maker movement, and elements of Design Thinking. These educational tools promote the protagonism of the student and enhance their capacities in the midst of a needy community, which mostly has little access to information technology and digital communication in their social contexts.

Keywords: design thinking ; digital inclusion; learning; maker culture; robotics; visual programming 


\section{Introducción}

\section{Educación básica y el aprendizaje de nuevas tecnologías}

La era del conocimiento presenta un nuevo desafío: la preparación de niños, jóvenes y adolescentes para una formación ciudadana y con perspectivas profesionales, quizás utilizando herramientas tecnológicas y prácticas pedagógicas que pueden ayudar al estudiante para la actual sociedad y su perspectiva hacia el futuro (Basile \& López, 2020).

La sociedad actual está inmersa en un entorno tecnológico, al igual que nuestros estudiantes, y esta percepción hace que las tecnologías cada vez estén más presentes y sean fundamentales para entender el futuro de la sociedad (Rodríguez, Ramírez \& Basile, 2016). Las instituciones educativas no pueden negar este hecho y deben seguir esta evolución constante. La tecnología está presente en nuestros televisores, en los frenos ABS de un coche, en los teléfonos smartphone, en un fax, en una contraseña de tarjeta bancaria y también en una infinidad de equipos que forman parte del día a día de las personas.

Sin embargo, en un entorno escolar son escasas las oportunidades tecnológicas, y es por esto que son necesarios estudios sobre los procedimientos que conducen a la ejecución y usos tecnológicos. El despertar en nuestros estudiantes surge del deseo de aprender, inspirarse en una clase más "conectada" y, por qué no, híbrida, donde se combinen diferentes estrategias didácticas y pedagógicas que los acerquen al uso adecuado de la tecnología disponible.

La robótica permite a los estudiantes tomar conciencia de la tecnología actual, desarrollar habilidades y catorce competencias, tales como: el trabajo de investigación, la capacidad crítica, el sentido del saber superar las dificultades en la resolución de problemas y el desarrollo del razonamiento lógico (Zilli, 2004).

Frente a todos estos avances tecnológicos, la sociedad del conocimiento y la nueva generación de estudiantes impulsan esta investigación para apoyar a la comunidad escolar, en respuesta a la pregunta de investigación de Blikstein (2018): ¿Cómo acercar experiencias innovadoras a los niños con menos oportunidades tecnológicas, de modo que sean acciones inclusivas que aporten una perspectiva de aprendizaje potencialmente idéntica a la que se lleva a cabo en las escuelas privadas y sociedades adineradas?

En consecuencia, el objetivo general de este estudio es presentar la enseñanza de la robótica pedagógica, en la cual el profesor usa metodologías activas y aplica conceptos y prácticas adaptables que permiten al estudiante experimentar y aplicar la cultura Maker y Design Thinking, así como posibilitar la inclusión digital con tecnologías en la educación básica en una comunidad pobre de la ciudad de São Paulo. 


\section{Marco teórico y conceptual}

\section{Metodologías activas de aprendizaje}

La innovación en el aprendizaje es una característica humana que se da para que sea posible alcanzar necesidades específicas y aumentar los conocimientos, de manera que produzca un mayor dinamismo útil en la educación formal y profesional (Zilli, 2004). Es así como la innovación en el aprendizaje se ha aplicado desde la perspectiva de metodologías activas de enseñanza-aprendizaje que consideran la era del conocimiento.

Diesel, Baldez y Martins (2017) describen el concepto de método activo de enseñanza y aprendizaje centrado en la figura del educando, donde el conocimiento se construye de forma colaborativa. En este punto conceptual, se hace una comparación con el método tradicional, donde el educador tiene un rol pasivo y el profesor el rol de transmisor de información. A partir de esta definición es posible delinear los principios que orientan las metodologías activas: el estudiante es el centro del proceso de aprendizaje, con autonomía, reflexión, problematización de la realidad, trabajo en equipo e innovación, en tanto que el profesor es un mediador, facilitador y asesor (Diesel et al., 2017).

Berbel (2011) utiliza la innovación en la forma en que aprendemos mediante una metodología activa para promover la autonomía de los estudiantes en el aula, con un enfoque específico de problematización, denominado "Arco de Maguerez", que es una construcción que permite al educando caminar a través de las siguientes fases: observación, identificación de puntos claves, teorización, hipótesis de acciones y aplicación, para que tenga la posibilidad de enfrentarse a la realidad y asociarla a los conocimientos previos pensando en una solución pertinente.

Gemignani (2013) destaca que cuando pensamos en el rol del estudiante en la praxis pedagógica es importante que tenga una postura activa, en situaciones de experiencia práctica. Esta perspectiva coincide con el trabajo de Berbel (2011), quien plantea la idea de utilizar la problematización para permitir al educando investigar y descubrir soluciones aplicadas a la realidad. De hecho, varios estudios han presentado las metodologías activas como una innovación en la práctica pedagógica que debe ser parte de la formación del profesor. En este sentido, la educación continua puede tener como consecuencia positiva la preparación de profesores capaces de optimizar sus prácticas en el aula, de tal manera que amplíen el acceso escolar y cultural de los educandos que no han tenido contacto cotidiano con los avances científicos y tecnológicos (Gemignani, 2013). Si bien este enfoque de problematización para resolver los problemas de la realidad se debe aplicar en las metodologías en la educación profesional (Barbosa \& De Moura, 2013), diversos estudios y proyectos desarrollados en Brasil evidencian que estas tecnologías se aplican de manera notable desde la educación primaria hasta la superior (Paiva et al., 2016). 
Al respecto, Moran (2018) destaca que cuando el educador utiliza las metodologías activas, es necesario que dicha acción sea convergente con los objetivos pedagógicos. En definitiva, la experimentación da innumerables nuevas posibilidades y con participación activa puede promover la formación de estudiantes más creativos.

\section{Cultura Maker}

El significado del término movimiento Maker (cultura Maker) se describe popularmente como una comunidad de aficionados, reparadores, ingenieros de piratería y artistas que crean y construyen proyectos utilizando herramientas, recursos y espacios, incluidos eventos, para que dichos hábitos se muestren y se compartan (Martin, 2015). En la visión educativa, se puede presentar como un camino de intervención pedagógica que podría operar en las escuelas con mayor atención, especialmente, a las visiones de la psicología del desarrollo, el diseño de interacción, el construccionismo y la educación progresiva (Blikstein, 2018).

El incentivo de la cultura Maker, "donde haces algo", es demostrar lo que has aprendido al hacer, de modo que se refuerza la evidencia del aprendizaje (Dougherty, 2012). La experiencia de la cultura Maker se pude comprobar mediante la participación de los educandos en la creación de proyectos para prototipos inmediatos y la participación de entornos culturales que fomenten la colaboración y el compartir (Niemever \& Gerber, 2015).

Dougherty (2012) también destaca el intercambio de ideas para resolver una serie de problemas, dentro de un espacio comunitario de colaboración e intercambio. El incentivo de ferias, eventos y otros tipos de integración social despierta el deseo de pensar para descubrir algo nuevo. Asimismo, sugiere que la vida se puede volver muy interesante cuando nos sumergimos en algo que no sabíamos hacer antes. Por ello, la cultura Maker es, además, la estructura de pensamiento con énfasis en la autonomía y la colaboración, que puede aportar un apoyo importante en el proceso de la enseñanza y el aprendizaje, considerando los diferentes tipos de contexto y disciplinas en la educación formal (Cohen et al., 2017).

\section{Design Thinking en la educación}

El término Design Thinkingse puede entender como un proceso analítico y creativo que permite al participante experimentar, crear y prototipar modelos, obtener retroalimentación sobre su desarrollo y plantear rediseños (Razzouk \& Shute, 2012). La aplicación del Design Thinking en el proceso de enseñanza y aprendizaje escolar puede cumplir la función de vínculo entre las estrategias pedagógicas culturales y las teorías de aprendizaje, porque este pensamiento está cerca de la forma constructivista actual y se adapta muy bien a proyectos interdisciplinarios que permiten el desarrollo de potenciales individuales (Scheer, Noweski \& Meinel, 2012).

La construcción de mapas mentales son un ejemplo del uso de la idea del proceso creativo y analítico desarrollado por un estudiante. Esta construcción en el aula puede reflejar el 
pensamiento del estudiante en un determinado dominio y evidenciar de qué manera está estableciendo relaciones con otros conceptos (Oxman, 2004). Los investigadores interesados en medir y respaldar el Design Thinking tienen grandes oportunidades no solo para realizar una amplia gama de estudios experimentales que pueden conducir a importantes descubrimientos, sino además para examinar los efectos del proceso del Design Thinking en varios resultados del aprendizaje (Razzouk \& Shute, 2012).

\section{Educación y las tecnologías digitales}

La Ley de Directrices y Bases (LDB) brasileña destaca la necesidad de crear políticas públicas que puedan ser implementadas para el uso y difusión de tecnologías digitales. Incluso se señalan metas en la educación básica para que se entiendan las tecnologías y sus implicaciones en la sociedad (Maia \& Barreto, 2012).

Zilli (2004) destaca que las tecnologías digitales pueden transformar la forma en que se enseña y aprende si se apuntan al desarrollo de habilidades y competencias, así como a la llamada articulación de los contenidos escolares, de tal manera que involucre una nueva forma de transformar, por un lado, la relación entre educadores y estudiantes, y, por otro, la cuestión de las tareas escolares.

La educación secundaria ha logrado usar las tecnologías digitales para mejorar los procesos de enseñanza y aprendizaje, lo cual se presenta como una solución importante en el contexto de las instituciones educativas actuales. Por ejemplo, los componentes curriculares de la educación secundaria, como la Física, fueron presentados a los estudiantes de manera adaptativa para que hubiera gran interés en el montaje de estructuras como montañas rusas, torres y pesas, e incluso en un carro que pudiera moverse desde la perspectiva de las leyes de la física y, principalmente, de la mecánica robótica (Bortolazza, Ribeiro \& Silva, 2014).

Basile y López (2020) analizan la importancia de realizar un trabajo educativo integrador en la educación técnica integrada con la educación básica. Estos trabajos son acompañados por educadores que promueven el trabajo en grupo para relacionar el uso de las tecnologías digitales con las necesidades que los estudiantes identifican en la comunidad donde viven. Las tecnologías empleadas están relacionadas con el uso de microprocesadores y computadores monoplaca, tanto en la tarea de ensamblar como de configurar dispositivos y programas que involucren el conocimiento de lenguajes y aspectos de gestión y seguridad de la información en este tipo de estudios técnicos integrados al bachillerato. En todos los niveles educativos brasileños, el uso de las tecnologías y las necesidades específicas de enseñanza y aprendizaje van acompañadas del uso intensivo de las nuevas tecnologías digitales, tanto para la comunicación sincrónica, como especialmente para que la educación pueda ir acompañada de la formación ciudadana y profesional. 


\section{La robótica y las experiencias educativas en Brasil}

La robótica pedagógica se puede ver como un proyecto escolar que involucra ciencia y tecnología aplicada a niños, educación primaria, media y superior. En este sentido, es importante observar algunas experiencias notables en el desarrollo de estas acciones de aprendizaje e inclusión digital de las comunidades brasileñas.

Brasil, con sus proporciones territoriales continentales, ha estado presentando proyectos con tecnología asociada a la robótica que son ejemplos de pedagogía proactiva, desde el norte hasta el sur del país. A continuación, se presentan algunos proyectos y se especifican las acciones realizadas que se acercan al informe de experiencias que se describen.

En la región sur, específicamente en la ciudad de Curitiba, Zilli (2004) presenta un trabajo analítico sobre cómo se pueden aplicar las tecnologías digitales en las escuelas primarias públicas y privadas mediante el uso de la robótica, como instrumento de aprendizaje que aporta una visión motivadora a los estudiantes. A modo de ejemplo, se cita el uso del internet y de tecnologías en entornos simulados que usan la ciencia en general como respuesta a la pregunta que se plantea el autor en el estudio: ¿Cómo el uso de la robótica en la enseñanza y el aprendizaje puede ser significativamente importante considerando las perspectivas constructivistas de la educación?

En la región nordeste, específicamente en el estado de Río Grande del Norte, el proyecto RoboEduc implementó una acción para lograr la inclusión digital de niños que en su experiencia común no tienen acceso a los recursos tecnológicos que tiene la sociedad de la era del conocimiento. En este estudio, los autores intentan trabajar en robótica educativa, tanto para enseñar informática y robótica, como para que sea complementaria en la enseñanza y el aprendizaje de componentes como las matemáticas y el portugués (Castro, 2008).

En la región norte, en el estado de Pará, también se asocia el uso de la robótica para ayudar en la enseñanza de las matemáticas. Específicamente, esta intervención se realiza en una comunidad "quilombola". En el contexto de una comunidad quilombola, los niños desfavorecidos tienen poca motivación para sus estudios debido a las dificultades, principalmente porque existe el gran problema de la deserción de los estudiantes como consecuencia de que muchos necesitan ayudar a sus padres en las tareas diarias de sus hogares. Por ello, la importante iniciativa de los autores, quienes usan un kit de robótica, concretamente el Lego Mindstorms Ev3, con cuestionarios prácticos que les permite obtener datos sobre la experiencia de los estudiantes con tecnologías de la información y la robótica (Meireles \& Mafra, 2016).

A pesar de los diversos proyectos de robótica educativa (pedagógica) que se llevan a cabo en todo el país, D’Abreu et al. (2013) señalan que no siempre fue así, pues las primeras iniciativas surgieron en el ámbito de proyectos universitarios y de centros de investigación lejos de la realidad, o en aulas con proyectos y diseños de importancia económica. Además de la perspectiva del uso de dispositivos comerciales, también es necesario entender que la 
robótica pedagógica se puede insertar en el contexto del aula, con recursos hardware y software abiertos y considerando la existencia de materiales de bajo costo (D'Abreu et al., 2013).

Recientemente, las tecnologías en el campo de la robótica están siendo apropiadas por la visión escolar. El término robótica pedagógica se utiliza para definir la instrumentalización de recursos, desde la figura de la educación infantil, primaria, secundaria y superior (Campos, 2011). La autora aporta su visión de los equipos para formación profesional, que han despertado interés debido a la percepción de una sociedad tecnológica. Actualmente, es posible observar que las tecnologías digitales asociadas a la robótica son empleadas en proyectos educativos, desde el primer año de primaria hasta la secundaria (Campos, 2011).

En la capital paulista, es importante destacar el proyecto de robótica con Sucata ('chatarra'), que involucra a niños y adolescentes necesitados para que sean incluidos digitalmente, para lo cual se usa la robótica como un medio de procesos de enseñanza y aprendizaje significativo para la comunidad en cuestión (Dias, 2019). La electrónica y los materiales que se desperdician tienen un nuevo destino cuando se incorporan al pensamiento computacional, la robótica, los lenguajes de programación y la electrónica (Dias, 2019). El gran desafío de esta labor es trabajar con recursos de bajo costo para construir los prototipos.

Específicamente, este estudio se enfoca en la relación entre las metodologías de aprendizaje activo, las tecnologías digitales en la educación y, especialmente, la aplicación de la robótica en desarrollar el potencial creativo de niños desfavorecidos para propiciar su inclusión digital.

\section{Currículo de la Ciudad de São Paulo}

La ciudad de São Paulo ha impulsado acciones para mejorar la educación básica y para ello creó el llamado Currículo de la Ciudad. Este nombre se deriva de su significado para orientar la alineación del plan de estudios de la ciudad de São Paulo con la Base Curricular Nacional Común (BNCC). Este currículo apunta a tres ejes fundamentales de la enseñanza en la ciudad: educación integral, equidad y educación inclusiva (Secretaria Municipal de Educação, 2017). En este estudio se describen los puntos significativos e importantes del proceso de enseñanza y aprendizaje con la robótica alineado con el concepto de equidad y educación inclusiva.

El concepto de equidad es aplicado por la escuela y por los profesores con la visión de formar estudiantes como seres íntegros, poderosos y autónomos, capaces de aprender. Asimismo, desde esta perspectiva se considera que los procesos educativos deben ser planificados y ejecutados de manera que consideren sus características y sus contextos, lo que puede representar un factor significativo en sus vidas (São Paulo, 2017).

La educación inclusiva es aplicada por las escuelas y por los educadores en acciones de respeto y apreciación de la diversidad y de las diferencias, siempre reconociendo las diferencias en el pensamiento y el aprendizaje de cada estudiante, y ciertamente desde una perspectiva de educación inclusiva, plural y democrática (São Paulo, 2017). 
El derecho a la educación es un punto destacado en el Currículo de la Ciudad en São Paulo porque enfatiza que se debe garantizar las condiciones y oportunidades para la formación de los estudiantes, para la formación de un ciudadano que pueda lograr una vida productiva a través del ejercicio de ciudadanía (São Paulo, 2017).

\section{Proyecto de robótica creativa: ordenanza y reglamento}

Es una iniciativa del Sistema de Educación Pública Municipal de la ciudad de São Paulo que tiene el nombre de Programa de Robótica Creativa. Fue creado por la Ordenanza N. ${ }^{\circ} 8699$, del 30 de diciembre de 2016, y se entiende como el uso crítico e innovador para adecuar la práctica docente en relación con demandas actuales de la sociedad. Según el artículo 2 de la ordenanza, el programa de robótica creativa tiene los siguientes objetivos: (1) difundir el uso de la robótica y el lenguaje de programación mediante la experimentación y la construcción del conocimiento; (2) generar oportunidades para que los estudiantes desarrollen habilidades lógico-matemáticas, de trabajo en grupo, habilidades motoras y de planificación de proyectos interdisciplinarios y que permiten el protagonismo del alumno, y (3) fortalecer la unidad educativa y recrear la cultura digital (São Paulo, 2016).

El alcance del programa de robótica creativa se trabaja desde la visión de la cultura Maker y el aprendizaje basado en resolver problemas y desafíos con un enfoque en estimular la libertad y la creación de toma de decisiones. Uno de los puntos más significativos de la inclusión digital es la construcción de prototipos con Kits estructurales y electrónicos, sin dejar de lado el aspecto humano importante para la formación continua del profesorado y el uso de las tecnologías de la información (São Paulo, 2016).

\section{Aplicación del modelo Profesor Orientador de Informática Educativa (POIE)}

El POIE es una designación que creó la Red Municipal de Educación de la ciudad de São Paulo para describir la función del profesor instructor de Informática Educativa, avalado por el consejo escolar, y que trabaja en espacios escolares de laboratorios de computación, con clases previstas en la organización escolar de todas las escuelas primarias (São Paulo, 2017).

\section{Materiales didácticos para enseñar robótica}

Los materiales didácticos para instrumentalizar la acción educativa se basan en un kit de robótica, el cual cuenta con un conjunto de componentes mecánicos de ensamblaje, ejes, motores y sensores. Además de esta parte mecánica, hay un microcontrolador que conecta sensores y motores a una computadora. Este tipo de estructura se basa en un entorno de robótica y mecánica permeado por la tecnología de la información, con sus elementos esenciales de hardware y software. Así, el hardware interactúa con la mecánica, la robótica, los lenguajes de programación y los programas para que las acciones de la robótica se ejecuten en el entorno real.

Específicamente, se utilizó el Kit de Robótica Atto Educacional para realizar acciones de mediación pedagógica con la planificación de la robótica pedagógica asociada a la intervención 
del educador en un ambiente de laboratorio de computación. En la figura 1 se muestra el Kit Atto Educacional con componentes electrónicos y partes para el contexto de la creación de elementos robóticos.

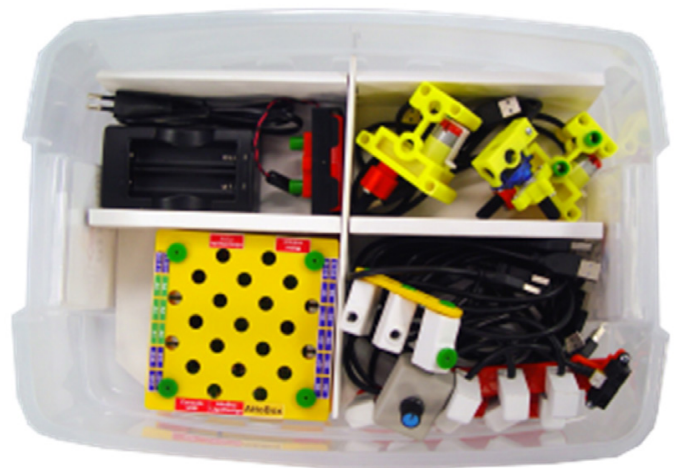

Figura 1. Conjunto de componentes electrónicos del Kit Atto Educacional. Fuente: Atto Educacional (s. f.).

\section{Metodología}

La investigación aplica el método experimental retroalimentado en educación (Fortunato, 2018), mediante el cual se estructuraron las estrategias didácticas que utilizaron los profesores desde la perspectiva de la enseñanza de tecnologías digitales y robótica para la inclusión digital y la mediación pedagógica en la educación primaria.

\section{Antecedentes y motivación}

Existen tres motivaciones extrínsecas que son importantes en el diseño del proyecto de robótica creativa y que forman parte de las iniciativas fundamentales de educación continua que desarrolla la División Pedagógica (DIPED) del SME (Secretaria Municipal de Educação):

1. La existencia de la cultura Maker o práctica se refiere a un movimiento creciente en el que las tecnologías digitales modernas contribuyen a la producción de artefactos físicos a través de la creación, la iteración, los intercambios y la autonomía (Cohen et al., 2017). Dentro de esta perspectiva existe una motivación para aprender a hacer, de manera que la robótica pedagógica puede hacer un aporte significativo en la realización de proyectos. Al respecto, Niemeyer y Gerber (2015) señalan que las comunidades de juego —donde la cultura Maker está presentepueden traer prácticas instructivas innovadoras que promueven una experiencia en el aula más colaborativa y participativa. 
2. La formación continua para profesores desde la Robótica JAM2017, celebrada entre el 28 y 29 de noviembre de 2017, aportó ideas y una visión sobre el uso de la robótica y las nuevas tecnologías. A este evento asistieron cerca de ochocientos estudiantes de escuelas municipales de la Ciudad de São Paulo, quienes experimentaron el mundo de la robótica con kits educativos. Este encuentro ofreció una nueva perspectiva a los estudiantes que nunca habían tenido contacto con las tecnologías, con la mecánica, la electrónica y la informática.

3. La tercera motivación surge después de visitar la red de laboratorios públicos, equipados con impresoras 3D, cortadoras laser, equipos de electrónica y robótica, denominada Fab Lab. La acción de formación continua en el Fab Lab de CEU Tres Puentes supuso una importante consolidación de conocimiento sobre el uso de impresoras de 3D y proyectos que se podrían desarrollar dentro del entorno escolar. En este orden, además de visitar las instalaciones, los estudiantes recibieron una formación sobre el uso de la impresora 3D y contaron con el apoyo institucional complementario en el tema de robótica pedagógica. Al finalizar, se destacó que ese espacio se puede utilizar para realizar nuevos proyectos.

\section{Epistemología para la acción}

La epistemología para la acción está guiada por el Programa de Robótica Creativa, instituido por la Ordenanza N. 8699 del 30 de diciembre de 2016, y el Currículo de la Ciudad. Ambos documentos fueron establecidos por el Departamento de Educación Municipal, con la autorización de la Alcaldía de São Paulo. Los estudios sobre la Cultura Maker en educación se enfocaron en el potencial de innovación de las tecnologías en las escuelas públicas de entorno comunitario, un desafío que debe ser afrontado por educadores y políticas públicas (Blikstein, 2018). El desarrollo de las acciones se presenta a continuación:

\section{Caracterización de la comunidad escolar}

Las acciones del proyecto de robótica creativa se realizaron en 2019 en la Escuela Municipal de Educación Primaria (EMEF) Capistrano de Abreu, en el barrio Itaim Paulista, un entorno caracterizado por la existencia de familias extremadamente pobres y socialmente excluidas debido a las condiciones socioeconómicas.

\section{Planeación}

La planificación de prácticas asociadas al uso de la robótica creativa también consideran las denominadas matrices de conocimiento, que destacan la correlación de los objetivos pedagógicos con aspectos de empatía y colaboración, recopilación cultural, pensamiento crítico, resolución de problemas, comunicación, autoconocimiento y autocuidado, autonomía y determinación, además de la apertura a la diversidad y la responsabilidad, sin dejar de lado la par- 
ticipación de los estudiantes inscritos en esta propuesta. En febrero de 2019 se elaboró la planificación para delinear el proyecto de robótica creativa del programa Más Educación São Paulo, que tiene como objetivo insertar la educación robótica para los estudiantes en el sistema educativo municipal.

El proyecto se llevó a cabo entre marzo y noviembre de 2019 con los estudiantes de la jornada tarde. Inicialmente, se planteó la posibilidad de ampliar el plan de estudios, pues en un principio solo se ofrecieron quince plazas a los estudiantes del ciclo interdisciplinario de primaria. Sin embargo, por la altas demanda, fue necesario abrir una segunda apertura para incluir a los estudiantes de la jornada de la mañana.

Los profesores ofrecieron varios cupos a los estudiantes, quienes llevaron una autorización de los padres con toda la información sobre el proyecto y sus respectivos cronogramas. Una vez que se cubrieron las vacantes, se inició el proyecto. Los estudiantes estaban muy emocionados porque era la primera vez que en la escuela se ofrecía el proyecto con una temática tan relevante para ellos y la sociedad.

A continuación, la figura 2 organiza las principales estrategias planificadas en el proceso de enseñanza y aprendizaje del proyecto de robótica creativa: inclusión digital, metodologías de aprendizaje activo, cultura Maker, Design Thinking y programación visual.

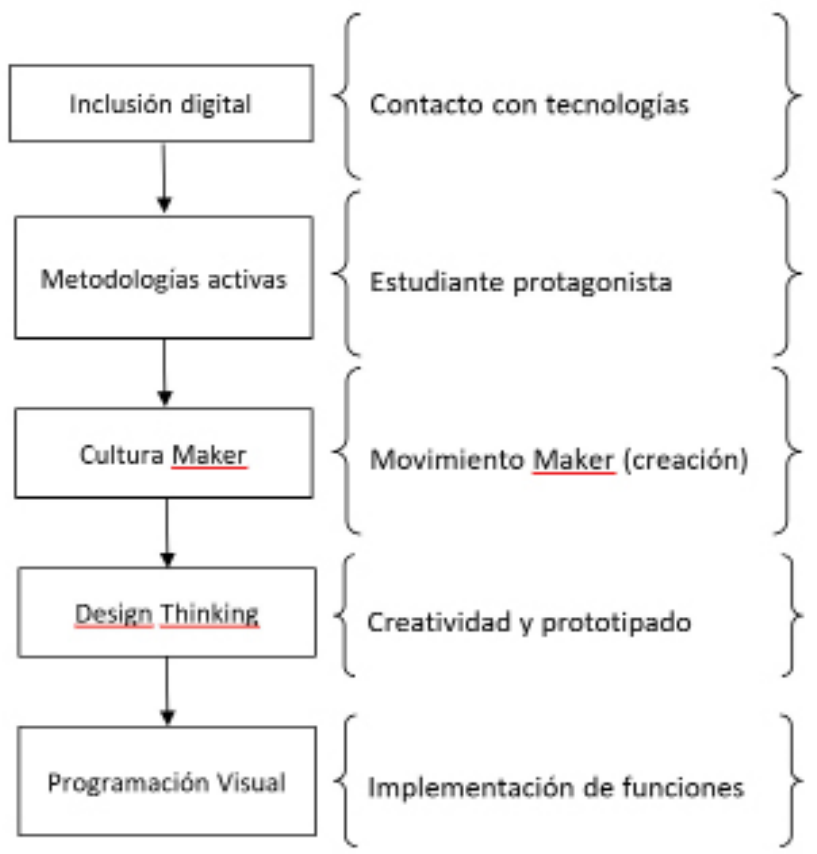

Figura 2. Estrategias fundamentales del proyecto de robótica creativa.

Fuente: elaboración propia. 
La tabla 1 describe las estrategias de acción del proyecto descritas anteriormente. Es importante mapear las acciones que fueron delineadas para implementar los siguientes pasos de la intervención pedagógica.

Tabla 1. Planeación para la intervención pedagógica

\begin{tabular}{|c|c|c|c|}
\hline Estrategias & Acciones & Objetivo & Materiales \\
\hline $\begin{array}{l}\text { Inclusión } \\
\text { digital }\end{array}$ & $\begin{array}{l}\text { Evaluación } \\
\text { diagnóstica. }\end{array}$ & $\begin{array}{l}\text { Identificar los conocimientos previos } \\
\text { sobre informática y robótica. }\end{array}$ & $\begin{array}{l}\text { Laboratorio de informática, } \\
\text { papel, bolígrafo y percepción } \\
\text { del profesor del perfil de los } \\
\text { estudiantes. }\end{array}$ \\
\hline $\begin{array}{l}\text { Metodologías } \\
\text { activas }\end{array}$ & $\begin{array}{l}\text { Rondas de } \\
\text { conversación. }\end{array}$ & $\begin{array}{l}\text { Comprender los pensamientos de } \\
\text { los niños a partir de la lectura sobre } \\
\text { informática y robótica. }\end{array}$ & $\begin{array}{l}\text { Folletos y materiales del kit de } \\
\text { robótica en el laboratorio de } \\
\text { computación. }\end{array}$ \\
\hline $\begin{array}{l}\text { Design } \\
\text { Thinking }\end{array}$ & $\begin{array}{l}\text { Lluvia de } \\
\text { ideas. }\end{array}$ & $\begin{array}{l}\text { Seleccionar ideas que se deban } \\
\text { trabajar para comprender la robótica y } \\
\text { la informática. }\end{array}$ & $\begin{array}{l}\text { Papel, bolígrafo, computadores } \\
\text { de laboratorio de investigación } \\
\text { del internet. }\end{array}$ \\
\hline $\begin{array}{l}\text { Design } \\
\text { Thinking }\end{array}$ & $\begin{array}{l}\text { Mapas } \\
\text { mentales. }\end{array}$ & $\begin{array}{l}\text { Crear mapas mentales sobre la } \\
\text { información adquirida para la lectura y } \\
\text { discusión en el laboratorio. }\end{array}$ & $\begin{array}{l}\text { Papel, bolígrafo, folletos y } \\
\text { materiales del kit de robótica. }\end{array}$ \\
\hline $\begin{array}{l}\text { Design } \\
\text { Thinking y } \\
\text { cultura Maker } \\
\text { en la inclusión } \\
\text { digital }\end{array}$ & $\begin{array}{l}\text { Creación de } \\
\text { prototipos. }\end{array}$ & $\begin{array}{l}\text { Modelar proyectos con piezas del Kit } \\
\text { Educativo Attos para pensar en el } \\
\text { diseño del elemento de robótica en el } \\
\text { que se trabajará. }\end{array}$ & $\begin{array}{l}\text { Kit de robótica para montaje y } \\
\text { contacto de los alumnos con las } \\
\text { piezas mecánicas. }\end{array}$ \\
\hline $\begin{array}{l}\text { Metodologías } \\
\text { activas y } \\
\text { cultura Maker } \\
\text { en la inclusión } \\
\text { digital }\end{array}$ & $\begin{array}{l}\text { Programación } \\
\text { visual. }\end{array}$ & $\begin{array}{l}\text { Utilizar enfoques tecnológicos para } \\
\text { programar mediante bloques lógicos } \\
\text { y activar funcionalidades mecánicas y } \\
\text { electrónicas a través de la tecnología } \\
\text { de la información. }\end{array}$ & $\begin{array}{l}\text { Computadoras de laboratorio, } \\
\text { kit de robótica de ensamblaje, } \\
\text { software de programación de } \\
\text { bloques visuales. }\end{array}$ \\
\hline
\end{tabular}

Fuente: elaboración propia.

\section{Intervención pedagógica}

La intervención pedagógica con los estudiantes se intentó construir a partir de los siguientes principios: colaboración, pensamiento reflexivo, construcción de información, cultura digital, protagonismo, autonomía e inventiva, que son útiles para trabajar con tecnologías para el aprendizaje que están impregnadas con estos principios.

\section{Evaluación diagnóstica}

En este estudio participaron estudiantes de $5 .^{\circ}$ y $6 .^{\circ}$ grados de la escuela primaria, quienes en su mayoría tenían poco dominio de la lengua portuguesa y de matemáticas, por lo cual el proyecto les ayudó en cierta manera a desarrollar estos conocimientos. Los estudiantes fueron seleccionados por sorteo, dado que el número de vacantes fue reducido en relación con el número de inscripciones. Inmediatamente después de completar el número de vacantes para 
el proyecto, se realizó el diagnóstico para conocer los conocimientos previos que tenían los estudiantes sobre robótica. Con este propósito, primero se pidió a los estudiantes que definieran el término robótica en una hoja de papel y luego se socializaron sus respuestas.

\section{Rondas de conversaciones sobre tecnologías digitales y robótica}

Las acciones de aprendizaje de robótica se iniciaron con un ciclo de conversación sobre las expectativas que se tenían del proyecto, lo que entendían sobre robótica y sus experiencias con las construcciones electrónicas. Luego se realizó la construcción de un panel donde los estudiantes definían con un término qué era robótica desde sus propias palabras y su propio vocabulario (figura 3). Al comienzo, con el fin de que tuvieran el primer contacto con la experiencia, se crearon oportunidades para que los estudiantes manipularan el equipo, se familiarizaran con su uso y aplicaran su creatividad ensamblando diversos objetos. De esta manera pudieron explorar sus potencialidades creativas, incluso sin la funcionalidad del Kit de Electrónica en ese momento. Posteriormente, se presentó el proyecto de robótica creativa, destacando los objetivos y el trabajo que se realizaría durante los encuentros presenciales. Además, se mostró la estructura del Kit Atto Educacional, con las partes estructurales y electrónicas.

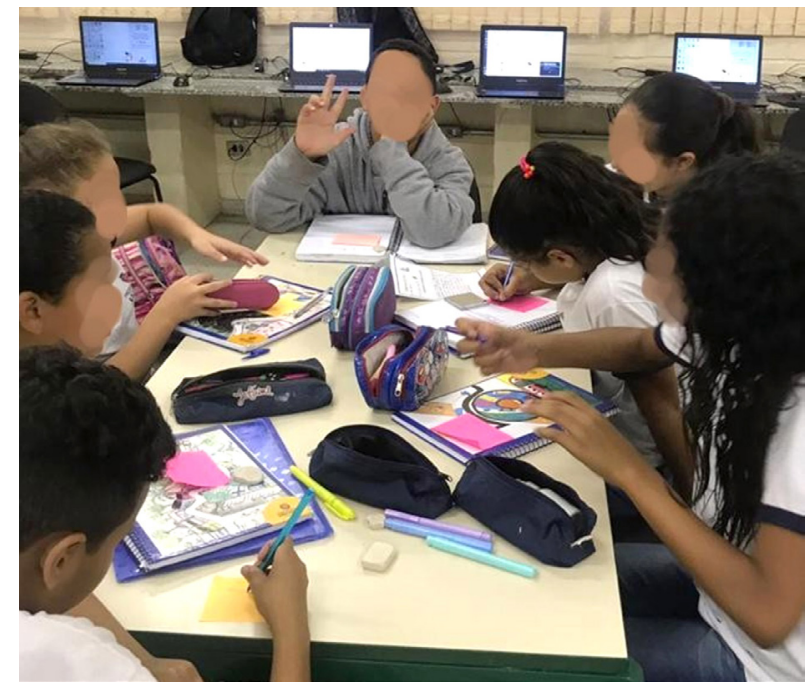

Figura 3. Ronda de conversación sobre robótica entre estudiantes.

Fuente: elaboración propia.

\section{Lluvia de ideas sobre aplicaciones robóticas}

Los estudiantes aprendieron a organizar sus ideas y pensamientos, aprendieron y mejoraron su creatividad exploratoria, sus habilidades y potenciales. A través de la pregunta de cómo mejorar la escuela mediante la robótica, los estudiantes reflexionaron sobre su realidad y bus- 
caron diferentes formas de mejorar aspectos de su comunidad escolar. Todos pensaron en un proyecto que podrían hacer para mejorar su día a día en el colegio y el de otros estudiantes, con lo cual observaron el potencial de creatividad que tenían y trabajaron en equipo para la solución de problemas.

\section{Mapas mentales sobre robótica}

En esta etapa, los estudiantes realizaron la lectura y estudio del folleto del Kit Atto Educacional, el cual explica cada componente para desarrollar las diferentes actividades propuestas, por ejemplo, el control de motor de corriente continua y el servomotor. Además, se aplicaron las metodologías activas para facilitar el aprendizaje de los estudiantes y se elaboraron mapas mentales como una forma de aprendizaje para corregir las técnicas de estudio: los estudiantes crearon los mapas mentales de lo que habían visto y escuchado de cada componente estudiado. Asimismo, también crearon otros mapas mentales en los que destacaban los componentes aprendidos en clase, de manera que el aprendizaje de los estudiantes se volvió innovador, con un aprendizaje más significativo en el que expresaron la organización de su pensamiento sobre un determinado tema.

\section{Design Thinking para la creación de prototipos}

A partir de los problemas que se identificaron en la lluvia de ideas y usando los principios del Design Thinking, los estudiantes plantearon soluciones posibles a los problemas escolares. Entre muchos de los temas que propusieron, el más relevante fue la inclusión de sus compañeros con escasos conocimientos de robótica (figura 4).

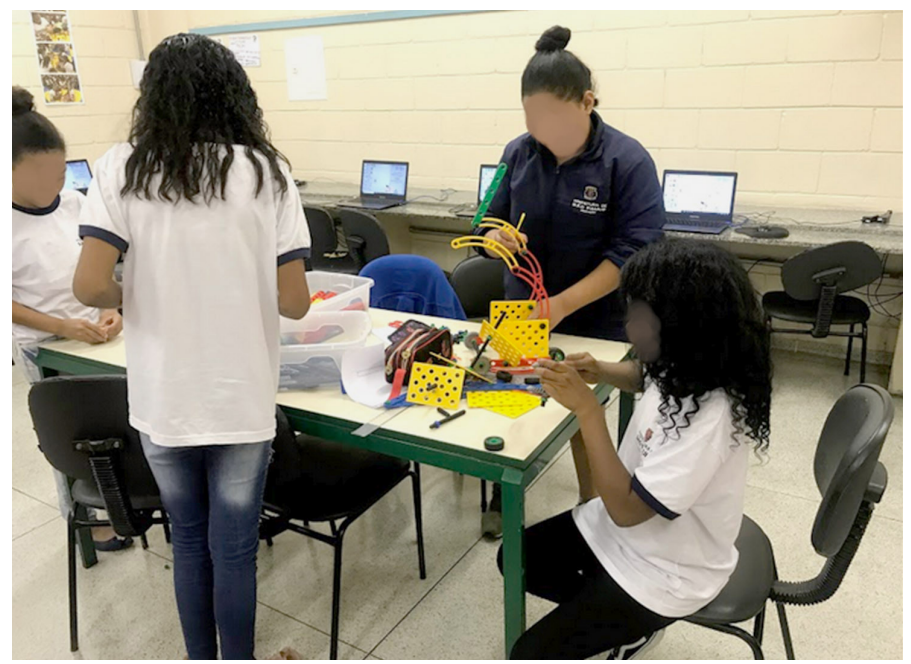

Figura 4. Actividad colaborativa de Design Thinking con el Kit Attos Educacional. Fuente: elaboración propia. 


\section{Programación visual de los prototipos creados}

La robótica creativa incluye el aprendizaje de la programación visual, que se utilizó para programar movimientos y acciones de prototipos robóticos desarrollados por los estudiantes a través del Design Thinking. Con este propósito se utilizó el software Ardublock ${ }^{\circledR}$ porque pere mite implementar funciones de movimiento de forma intuitiva, con acciones de arrastre y conexiones. La figura 5 muestra el entorno del desarrollo integrado (IDE) de Ardublock ${ }^{\circledR}$, que tiene este principio de programación visual con bloques encadenados. Este sistema permite una secuencia lógica programática, la cual se incorporó para que los estudiantes programaran los objetos ensamblados desde la perspectiva del Design Thinking en los momentos de actividad con POIE en un laboratorio de computación.

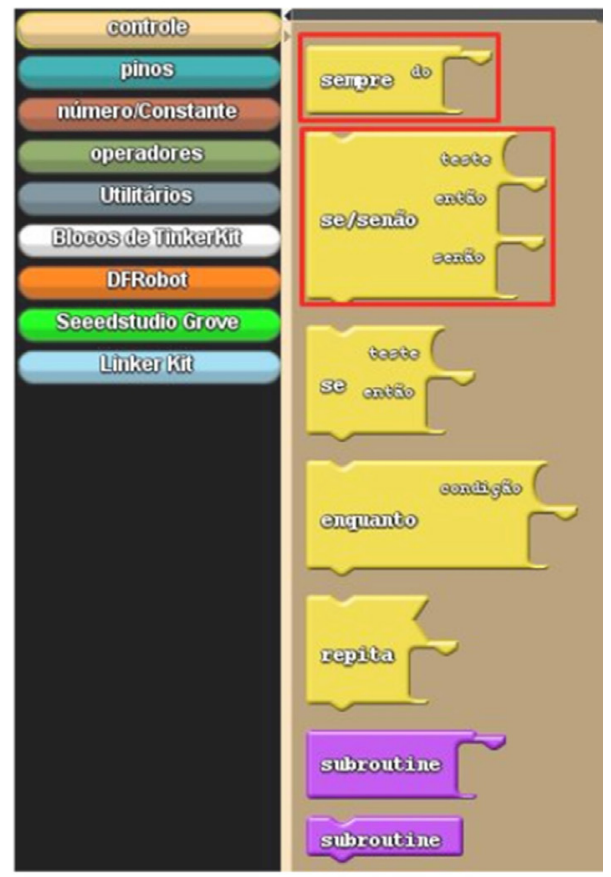

Figura 5. IDE Ardublock ${ }^{\circledR}$ con estructura de bloques de programación. Fuente: Atto Educacional (s. f.).

\section{Resultados}

\section{Formación en la clase de robótica}

La formación de clases de robótica creativa se llevó a cabo con matrículas de estudiantes de primaria, de 10 a 11 años de edad. Se crearon dos grupos para llevar a cabo el proyecto en 2019. En el Grupo 1 participaron 15 estudiantes y en el Grupo 2 participaron 13, con un total de 28 estudiantes inscritos en el proyecto de robótica creativa. 
Los alumnos aprendieron el lenguaje de programación visual Ardublock ${ }^{\circledR}$, que se asoció con Arduino (Robotics Kit - Educacional Act), y diseñaron estructuras y prototipos con la cultura Maker y metodologías activas. Además los desarrollaron con autonomía y reflexión crítica para la resolución de problemas en el uso de la tecnología.

\section{Talleres de aprendizaje de tecnología y robótica}

En diciembre de 2019, los estudiantes de ambos grupos de robótica participaron en el Festival de Inventiva y Tecnologías para el Aprendizaje. El Festival se desarrolló en dos jornadas: en la mañana y en la tarde. El docente trabajó con el rol de POIE para que los estudiantes participaran en los talleres, en los cuales los alentaron a tener nuevos contactos con las tecnologías digitales y el uso de la robótica a través de los siguientes temas: Ser Marker; Terror de quebrada-experiencia de creatividad; Robótica creativa; El arte de HQ; Creando con Scratch; Tesoro perdido del campamento de pajitas; Modelar con Tinkercard; JAM, y Moldes de silicona.

\section{Recursos audiovisuales sobre la robótica creativa}

En las actividades de aula se crearon recursos audiovisuales (figura 6) usando la guía del POIE y la perspectiva aplicada del Design Thinking para consolidar los conceptos de robótica. Se creó una parodia de la canción Despacito para transmitir el mensaje de la robótica creativa a colegas y amigos de la comunidad.

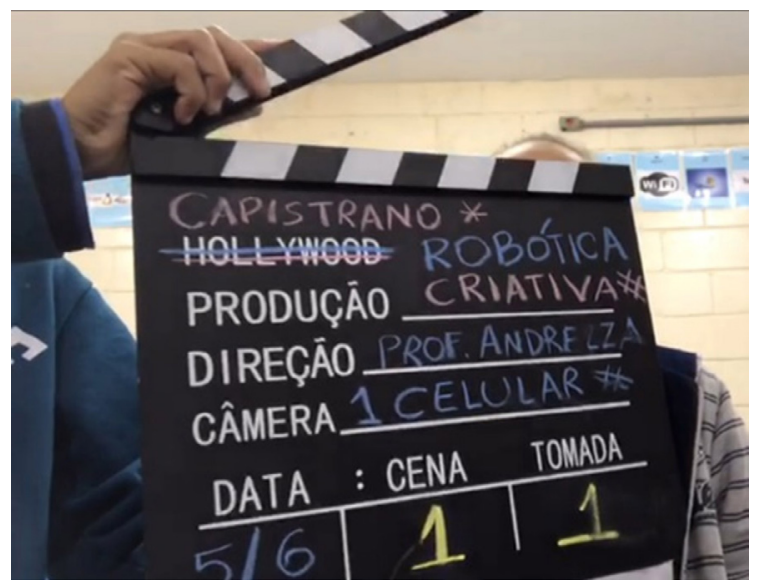

Figura 6. Actividad de creación de recursos audiovisuales.

Fuente: elaboración propia.

\section{Prototipos funcionales con robótica y programación visual}

Los prototipos que desarrollaron los estudiantes durante las clases tomaron la forma de objetos mediante las actividades de Design Thinking. A partir de esta estructura en objetos físicos fue posible trabajar con los elementos de robótica: partes mecánicas, estructura y programación visual con desarrollo integrado (IDE) en Ardublock ${ }^{\circledR}$ para lograr el funcionamiento de las partes. 
La figura 7 muestra el trabajo que realizan los estudiantes, en el cual usan la programación visual en un objeto para lanzar una pelota de ping pong. A partir de la comprensión de la relación entre la robótica y la tecnología de información, hacen la acción de conectividad de Atto Educacional Kit, notebook y Ardublock ${ }^{\circledR}$ IDE para que su prototipo se vuelva funcional. Es decir, la programación visual le permite llevar a cabo la acción de lanzamiento de la pelota.

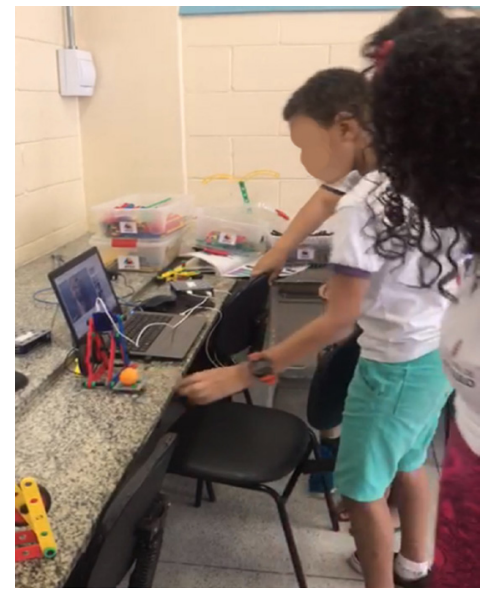

Figura 7. Estudiantes realizan programación visual del prototipo.

Fuente: elaboración propia.

La figura 8 muestra un par de estudiantes haciendo la programación visual y comprendiendo las acciones entre IDE Ardublock ${ }^{\circledR}$ y los movimientos mecánicos del objeto. Los estudiantes crearon un objeto robótico que representa la forma y la acción de un tucán que se mueve en un entorno natural. Este ejercicio destaca las acciones protagonizadas por los estudiantes con el prototipo objeto, la mecánica y las conexiones de la robótica y las tecnologías de información en un pensamiento creativo.

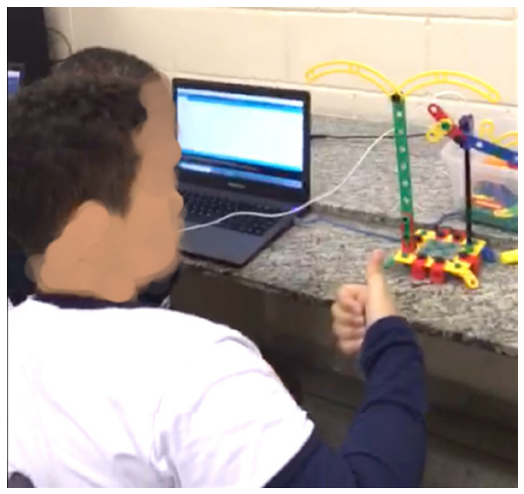

Figura 8. Estudiantes en acción experimental y de programación visual. Fuente: elaboración propia. 


\section{Impresión 3D}

La impresora 3D fue utilizada por los estudiantes en la clase del proyecto de robótica creativa con la idea que estuvieran en contacto con la tecnología digital. Con los recursos del entorno escolar fue posible crear un proyecto de llavero simple, que fue diseñado utilizando el software Tinkercard y desde la creación del proyecto. Todo este proceso se realizó con la supervisión del POIE (profesor mediador), observando y atendiendo todas las medidas de seguridad.

Esta experiencia propició el protagonismo de los estudiantes mediante el contacto con las tecnologías, la observación y, especialmente, la creación de un objeto real dentro del momento de creatividad del proyecto de inclusión digital. Esta acción fue importante porque los estudiantes pensaron de manera colaborativa cómo contribuir con sus compañeros con necesidades educativas. Todos querían ensamblar equipos de tecnología de asistencia utilizando la impresora 3D (figura 9). Posteriormente, los estudiantes y profesores buscaron entender qué significaba la tecnología de asistencia y el uso de la impresora 3D para poner en marcha el proyecto, para lo cual utilizaron el conocimiento de la robótica como eje fundamental del plan de acción.

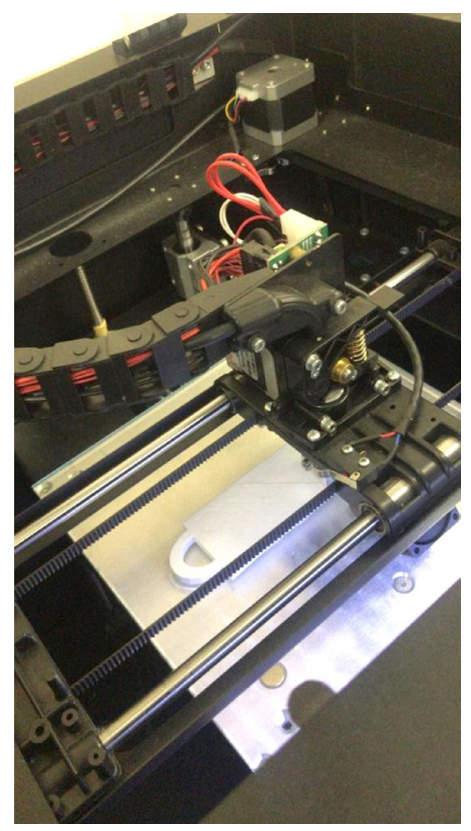

Figura 9. Impresora 3D usada por los estudiantes de los dos grupos.

Fuente: elaboración propia.

\section{Discusión}

Los resultados de esta investigación se basan en un estudio experimental que cumplió los objetivos planeados de la intervención pedagógica, para lo cual se asociaron estrategias, 
acciones, objetivos y uso de materiales que ayudaron a desarrollar el proceso de enseñanza y aprendizaje.

A continuación, se discutirá la importancia de los resultados y las opiniones de los autores de la literatura citada en este trabajo, para esbozar los aportes desde la perspectiva de la inclusión digital, las metodologías de aprendizaje activas, la cultura Maker, el Design Thinking y la programación visual del proyecto de robótica creativa inclusiva en EMEF Capistrano De Abreu.

\section{Inclusión digital de niños y adolescentes en comunidades de escasos recursos}

El cuestionamiento central de Blikstein (2018) trae una provocación a los educadores y al sistema educativo de las naciones: ¿Cómo generar las mismas oportunidades de aprendizaje para las comunidades pobres, enfatizando la importancia de la inclusión? Para lograrlo, la inclusión debe abarcar a los adultos, las personas de la tercera edad (Basile \& López, 2016; Marioto \& Basile, 2020), los jóvenes y, especialmente, la educación primaria.

Si bien en el desarrollo de las tecnologías digitales en diferentes entornos del sector productivo, empresarial y científico aún existen muchas acciones y políticas públicas, en educación es necesario trabajarlas en comunidades necesitadas. Al respecto, Maia y Barreto (2012) citan la Ley de Base y Lineamientos Educativos de Brasil (LDB), la cual destaca como una acción importante la existencia de políticas públicas que puedan brindar oportunidades para que los estudiantes experimenten el uso de las tecnologías y que la escuela pueda cumplir su papel de difundir el conocimiento e información sobre las tecnologías digitales.

Esta investigación enfatizó la aplicación de las políticas públicas en educación de la ciudad de São Paulo con la planificación de objetivos educativos. En este sentido, se considera que la creación del Programa de Robótica Creativa, instituido por la Ordenanza N. 8699, del 30 de diciembre de 2016, y el Currículo de la Ciudad posibilitan el proceso de inclusión digital, pues motivan a los estudiantes a acceder a materiales informáticos en un microcosmos de cibercultura (el laboratorio de computación) y el Kit de Atto Educacional.

Durante el desarrollo de las acciones con los estudiantes de la comunidad periférica se identificó que apenas existe acceso a tecnologías digitales, ya sean permeadas por robótica, computadoras o microcomputadoras.

\section{El estudiante protagonista desde la perspectiva de metodologías de aprendizaje activo}

El estudiante como centro del aprendizaje es uno de los principales actores en la aplicación de prácticas de metodologías activas (Diesel et al., 2017). Así mismo, dentro de la perspectiva del proyecto de robótica creativa, el microcosmos de la sala y la intervención del PoIE aportan significativamente a la valorización del proyecto.

Los resultados demostraron que la praxis pedagógica posibilitó aspectos importantes, como la postura activa y la práctica experiencial (Gemignani, 2013) de los estudiantes. De la misma manera, se debe destacar que el desarrollo de las estrategias y las acciones planifica- 
das, así como la construcción del plan del proyecto asociado a metodologías de aprendizaje activo —con la delimitación de los objetos pedagógicos recomendados por Moran (2018) — fueron parte fundamental del éxito de las acciones creadas para construir un ambiente propicio para el uso de estas metodologías en el proceso de enseñanza.

La motivación que tuvieron los estudiantes por leer los materiales y el manual del kit de robótica crearon la oportunidad para relacionar la lectura con la escritura, así como la lectura con la interpretación para superar las dificultades de aprendizaje en el idioma portugués. El uso del lenguaje de programación, incluso de alto nivel, exige que el estudiante estructure la lógica del pensamiento (razonamiento lógico-matemático) y la organización sintáctica en la escritura de comandos para controlar modelos robóticos. En este sentido, esta intervención coincide con la postura de Neto et al. (2015), quienes consideran que el desarrollo de la interdisciplinariedad en la robótica es una herramienta eficiente para el aprendizaje.

\section{El papel del educador mediador de la cultura Maker}

La aplicación del movimiento Maker se evidencia en las acciones que fomentan el uso de tecnologías, la participación en eventos, la creación de espacios y el desarrollo práctico de proyectos educativos (Martin, 2015). Al respeto, se debe resaltar que en este proyecto se movilizaron estrategias y acciones pedagógicas en la comunidad, en línea con el pensamiento de Blikstein (2018), para quien existe una asociación entre el diseño de la interacción y la educación progresiva. En este sentido, el proyecto potenció el rol del educador mediador de la cultura Maker para que el estudiante viviera experiencias en el entorno escolar tecnológico con el laboratorio de computación y los materiales didácticos, incluidos los manuales de instrucciones y los folletos del kit de robótica. En consecuencia, se puede afirmar que esta experiencia fortalece la evidencia del aprendizaje (Dougherty, 2012) a través de los prototipos funcionales que produjeron los estudiantes, mediante los cuales participaron en la cultura Maker (Hacer-lo).

\section{Design Thinking para la ideación y creatividad de prototipos con programación visual}

El Design Thinking se puede aplicar con diversas actividades que ayuden al estudiante a concebir lo que quiere hacer y a formularlo a través de ideas, con lo cual se estimula el desarrollo de su creatividad. Siguiendo esta orientación, el proyecto estructuró un espacio en el entorno escolar para concebir estas ideas mediante acciones como los círculos de conversación, la Iluvia de ideas, los mapas mentales y los prototipos. Durante las rondas de conversación se crearon espacios colaborativos para las clases, donde los estudiantes expresaban sus conocimientos previos sobre lo que es la robótica.

Dado que en esta propuesta el estudiante es el centro del aprendizaje, era muy importante escuchar y resaltar la lluvia de ideas, que fueron importantes para organizar el pensamiento del estudiante sobre los conceptos fundamentales de la robótica y las tecnologías de la información y la comunicación digital. De esta manera, las acciones de los circuitos de 
conversación y las lluvias de ideas contribuyeron a que los estudiantes establecieran la relación entre los conceptos importantes (Oxman, 2004), al tiempo que permitieron al profesor mediador crear nuevas oportunidades de ideación y creatividad en el padrón que se necesita.

Finalmente, hay que tener en cuenta que la creación de prototipos de objetos robóticos, como los realizados en este proyecto, son eventos de pensamiento de diseño significativo que evidencian un aprendizaje activo basado en modelos (Razzonk \& Shute, 2012) y permiten que los logros sean efectivos y no solamente orientados para el objeto funcional, además de que resaltan el potencial del estudiante (Scheer et al., 2012) mediante su participación en robótica creativa.

\section{Conclusiones}

Este proyecto de robótica creativa soporta una fuerte investigación sobre el uso de la cultura Maker y contribuye a las futuras intervenciones relacionadas con la evolución de la robótica pedagógica inclusiva, de manera que brinda una nueva visión sobre posibles experiencias de aprendizaje de tecnologías para los estudiantes menos favorecidos.

Esta sinergia de las acciones con los resultados observados se sustenta en los ejes de trabajo asociados con las estrategias y las acciones realizadas. Entre estas se destacan la enseñanza de la robótica con inclusión digital para el apoyo pedagógico de la escuela pública y la adecuación de la infraestructura de los laboratorios y de los equipos para que posibiliten la experiencia del estudiante como protagonista del proceso.

Las metodologías activas de aprendizaje que se aplicaron en el espacio educativo del proyecto pedagógico POIE destacaron el papel activo y participante del estudiante. Asimismo, la perspectiva de la cultura Maker permitió organizar el ambiente escolar y estructurarlo como un lugar agradable para el aprendizaje, de manera que los deseos de aprender y crear fueron posibles gracias a que los estudiantes trabajaron con la mentalidad de construir objetos a partir de sus propias ideas y se destacó en todo momento su potencial.

El Design Thinking proporcionó al estudiante y al profesor herramientas creativas para desarrollar ideas en el mundo analógico y digital para construir objetos robóticos. Por su parte, la programación visual permitió a los participantes concebir las acciones que realizarían los elementos de los objetos robóticos que desarrollaron, con lo cual aportó significativamente a elaborar una percepción de la creación y a dar vida a los objetos creados.

Finalmente, se concluye que todas estas estrategias asociadas a las acciones de intervención pedagógica organizada por las políticas públicas de educación pueden potenciar la enseñanza de la robótica en comunidades desatendidas y aumentan el impacto de la cultura Maker inclusiva con robótica creativa.

\section{Agradecimientos}

Los autores desean agradecer a la Secretaria Municipal de Educação de São Paulo, al Instituto Federal de Educação e Tecnologia Campus São Paulo Pirituba, y a la Universidad Militar Nueva Granada por su apoyo en la realización de este artículo. 


\section{Declaración de divulgación}

Los autores declaran que no existe ningún potencial conflicto de interés relacionado con el artículo.

\section{Financiamiento}

Los autores no declaran fuente de financiamiento para la realización de este artículo.

\section{Sobre los autores}

Andrezza da Costa Rodrigues Basile es pedagoga de la Universidad Braz Cubas. Especialista en Educación Inclusiva y Formación en Educación a Distancia (aprendizaje a distancia). Trabaja como docente en la red de educación municipal de la ciudad de São Paulo.

https://orcid.org/0000-0002-1411-4864 - Contacto: andrezza.rodrigues@sme.prefeitura.sp.gov.br

Felipe Rodrigues Martinez Basile es ingeniero en Sistemas de Información. Magíster y doctor en Ingeniería Biomédica de la Universidad de Mogi das Cruzes. Especialista en Seguridad de la Información. Es profesor e investigador en Redes Informáticas en el IFSP - Campus PTB, Brasil. Realiza investigaciones multicéntricas sobre seguridad de la información en Suramérica.

https://orcid.org/0000-0002-0404-4807 - Contacto: felipe.basile@ifsp.edu.br

Leonardo Juan Ramírez López es ingeniero electrónico y especialista en instrumentación electrónica. Magíster en Ingeniería de Sistemas de la Universidad Nacional de Colombia y doctor en Ingeniería Biomédica de la Universidad de Mogi das Cruzes de São Paulo (Brasil). Profesor e investigador senior de la Universidad Militar Nueva Granada, Bogotá, D. C., Colombia. https://orcid.org/0000-0002-6473-5685 - Contacto: leonardo.ramirez@unimilitar.edu.co

\section{Referencias}

Atto Educacional. (s. f.). Atto Educacional. https://attoeducacional.com.br/

Barbosa, E. F., \& De Moura, D. G. (2013). Metodologias ativas de aprendizagem na educação profissional e tecnológica. Boletim Técnico do Senac, 39(2), 48-67.

Basile, F. R. M., \& Ramírez López, L. J. (2020). Estrategia formativa en defensa digital para adolescentes: experiencia en el Instituto Federal de São Paulo. Revista Científica General José María Córdova, 18(30), 271-287. https://doi.org/10.21830/19006586.579

Berbel, N. A. N. (2011). As metodologias ativas e a promoção da autonomia de estudantes. Semina: Ciências Sociais e Humanas, 32(1), 25-40.

Blikstein, P. (2018). Maker movement in education: History and prospects. Handbook of Technology Education, 419-437.

Bortolazza, C., Ribeiro, D. J., \& Silva, W. D. O. (2014). O uso da robótica educacional em aulas práticas de física no ensino médio. Anais do IV Simpósio Nacional de Ensino de Ciência e Tecnologia, 1-9.

Campos, F. R. (2011). Currículo, tecnologias e robótica na educação básica. PUC. São Paulo.[GS Search].

Castro, V. G. D. (2008). RoboEduc: Especificação de um Software Educacional para ensino da Robótica às crianças como uma ferramenta de inclusão digital[tesis de maestría, Universidade Federal do Rio Grande do Norte]. Repositorio Institucional. https://repositorio.ufrn.br/jspui/handle/123456789/15217

Cohen, J., Jones, W. M., Smith, S., \& Calandra, B. (2017). Makification: Towards a framework for leveraging the maker movement in formal education. Journal of Educational Multimedia and Hypermedia, 26(3), 217-229. 
D’Abreu, J. V. V., Ramos, J. J., Mirisola, L. G., \& Bernardi, N. (2013, octubre). Robótica educativa/pedagógica na era digital. En // Congresso Internacional T/C e Educação (vol. 15). http://www.informaticaeducativa. com.br/artigos/Aula\%204/ROB\%C3\%93TICA\%20EDUCATIVAPEDAGOGICA\%20NA\%20ERA\%20DIGITAL.pdf

Dias Garofalo, D. D. (2019). Robótica com sucata. Revista Brasileira de Pós-Graduação, 15(34), 1-21.

Diesel, A., Baldez, A. L. S., \& Martins, S. N. (2017). Os princípios das metodologias ativas de ensino: uma abordagem teórica. Revista Thema, 14(1), 268-288.

Dougherty, D. (2012). The maker movement. Innovations: Technology, Governance, Globalization, 7(3), 11-14.

Fortunato, I. (2018). Método (s) de Pesquisa em Educação. São Paulo. Edições Hipótese, 5(1), 37-50.

Gemignani, E. Y. M. Y. (2013). Formação de professores e metodologias ativas de ensino-aprendizagem: ensinar para a compreensão. Fronteiras da Educação, 1(2).

Maia, D. L., \& Barreto, M. C. (2012). Tecnologias digitais na educação: uma análise das políticas públicas brasileiras. EFT: Educação, Formação \& Tecnologias, 5(1), 47-61.

Moran, J. (2018). Metodologias ativas para uma aprendizagem mais profunda. http://www2.eca.usp.br/ moran/wp-content/uploads/2013/12/metodologias_moran1.pdf

Martin, L. (2015). The promise of the maker movement for education. Journal of Pre-College Engineering Education Research (J-PEER), 5(1), 4.

Marioto, R. R., \& Basile, F. R. M. (2020). Escrita para terceira idade com o uso de tecnologias digitais: relato de experiência. Revista Internacional de Formação de Professores, 5, 1-19

Meireles, J. C., \& Mafra, J. R. S. (2016). A robótica como técnica de aprimoramento no ensino da Matemática no Quilombo de Peafú-Monte Alegre/Pará. Encontro Nacional de Educação Matemática (ENEM), 1-11.

Neto, R. P. B., Santana, A. M., Rocha, D. P., \& Souza, A. (2015, octubre). Robótica na educação: uma revisão sistemática dos últimos 10 anos. En Brazilian Symposium on Computers in Education (Simpósio Brasileiro de Informática na Educação-SBIE) (vol. 26, n. ํ. 1. p. 386). http://dx.doi.org/10.5753/cbie.sbie.2015.386

Niemeyer, D. J., \& Gerber, H. R. (2015). Maker culture and Minecraft: implications for the future of learning. Educational Media International, 52(3), 216-226.

Oxman, R. (2004). Think-maps: teaching design thinking in design education. Design Studies, 25(1), 63-91.

Paiva, M. R. F., Parente, J. R. F., Brandão, I. R., \& Queiroz, A. H. B. (2016). Metodologias ativas de ensino-aprendizagem: revisão integrativa. SANARE-Revista de Políticas Públicas, 15(2). https://sanare. emnuvens.com.br/sanare/article/view/1049/595

Razzouk, R., \& Shute, V. (2012). What is design thinking and why is it important? Review of Educational Research, 82(3), 330-348.

Rodríguez, A. B., Ramírez, L. J., \& Basile, F. R. (2016). Percepción social de las tecnologías electromagnéticas. Información Tecnológica, 27(6), 227-236.

Secretaria Municipal de Educação. Coordenadoria Pedagógica. (2017). Currículo da Cidade: Ensino Fundamental: Tecnologias para Aprendizagem. SME/COPED.

Secretaria Municipal de Educação. (2016). SME N. ${ }^{\circ} 8.699$ de 30 de dezembro de 2016. Diário Oficial da Cidade de São Paulo. http://legislacao.prefeitura.sp.gov.br/leis/portaria-secretaria-municipal-de-educacao-8699-de-30-de-dezembro-de-2016//consolidado

Scheer, A., Noweski, C., \& Meinel, C. (2012). Transforming constructivist learning into action: Design thinking in education. Design and Technology Education: An International Journal, 17(3), 8-19.

Zilli, S. D. R. (2004). A robótica educacional no ensino fundamental: perspectivas e prática [tesis de maestría, Universidade Federal de Santa Catarina]. Repositorio Institucional. https://repositorio.ufsc.br/ bitstream/handle/123456789/86930/224814.pdf?sequence=1\&isAllowed=y 